\title{
Security Analysis of Embedded HVDC in Transmission Grids
}

\author{
Marco Giuntoli and Susanne Schmitt
}

\begin{abstract}
Security-constrained optimal power flow is widely used by grid operators during their short-term operations. Possible contingencies (e.g. outages of lines, generators) analyzed during this phase may be very critical for the stability of the power system. Thus, a simplified or reduced approach might not be desired. Moreover, the presence of embedded high-voltage direct current (HVDC) links (i.e. the DC link is done between two or more synchronous AC nodes) increases the degrees of freedom of the physical problem and thus an economically better working point for the system can be reached. To do this, a coordinated control between the AC and DC systems is required using one single stage of optimal power flow including the security assessment. In this work the SC-OPF (Security-Constrained Optimal Power Flow) approach is used to analyse the effects of contingency cases within the DC system.
\end{abstract}

Keywords Optimal power flow - Security-constrained optimal power flow · Embedded HVDC · N-1 criterion

\section{Introduction}

There is a world-wide trend towards increasing the share of renewable energy sources for electricity generation. Since the yield of renewable energy sources like e.g. solar and wind power is time-varying and depends on geographic location there is an increasing demand for large-scale power transfer over long distances. In recent years there have been more and more high-voltage direct current (HVDC) links for power transmission commissioned and more are yet to be planned and installed. In [1] an extensive overview of the advantages of DC technology for power transmission is given. For instance, in Germany there are several embedded HVDC

\footnotetext{
M. Giuntoli · S. Schmitt $(\bowtie)$

ABB Corporate Research Center Germany, Ladenburg, Germany

e-mail: marco.giuntoli@de.abb.com; susanne.schmitt@de.abb.com

(C) The Author(s) 2020

V. Bertsch et al. (eds.), Advances in Energy System Optimization, Trends in Mathematics, https://doi.org/10.1007/978-3-030-32157-4_2
} 
links (i.e. they connect synchronous AC nodes) planned in order to transfer wind power from the north to the high load regions in the south, see [2].

Optimal power flow is a widely discussed optimization problem to determine the optimal operation of controllable equipment in a power grid, see e.g. [3, 4]. For transmission grid operation the so-called $N-1$ criterion as defined in [5] is a requirement. It means that for any single outage of a grid component (e.g. a generator, a line or a power transformer) the remaining grid must be able to operate safely within its limits. This leads to security-constrained optimal power flow problems (SC-OPF). For this class of problems an extensive overview is provided in [6]. SC-OPF problems can be formulated either in the preventive way, i.e. there is a single set of control variables that has to be feasible in all considered contingency cases or in the corrective way, i.e. for each contingency case there may be a different set of control variables, and their values can be reached by short-term control actions when a contingency case is taking place, see [7].

Optimal power flow formulations for hybrid AC/DC grids including security constraints are presented e.g. in [1, 8] and [9]. In [10] a corrective SC-OPF methodology is employed to investigate the capabilities of embedded HVDC systems to compensate for contingency cases in the AC system and thus to reduce redispatch by generators.

In this work we use SC-OPF to study the effects of the $N-1$ criterion with emphasis on contingency cases within the embedded HVDC systems. To our knowledge the effects of $N-1$ cases inside the DC systems have not yet been studied extensively in the literature. As in [10] we focus on the capabilities of the grid components and in particular the embedded HVDC systems to compensate for contingency cases and to avoid generator redispatch.

This work is structured as follows: in Sect. 2 we present the mathematical model for SC-OPF for hybrid AC/DC grids. Then, in Sect. 3 we describe the case study grid and scenario used as well as the implementation of the approach (Sect. 4). The case study results are presented and discussed in Sect. 5, and we give conclusions and suggestions for future work in the final Sect. 6.

\section{The Mathematical Model}

The mathematical model of the SC-OPF is used to perform a steady-state optimization for hybrid AC/DC grids, where the two types of grids are coupled via a set of power converters. The model is formulated such that just one single optimization problem can be used to solve the AC and DC grids simultaneously; this means in particular that no iterative algorithm is needed to reach the final solution.

The model is written to address one-phase electric systems, i.e. it can evaluate a mono-phase system or a multiphase system but in the latter case the system must be balanced and symmetric to consider only the positive sequence (Fortescue transformation). 
All the variables and equations are expressed in complex polar form and they are differentiable and continuous. This means that discrete variables (e.g. limited number of tap changer positions or discontinuous active power range for the generators) are not considered. The set of variables are split into two parts: control (or independent) variables $u$ representing power setpoints (active and reactive) for controllable devices as well as tap changer and phase changer positions for power transformers. State (or dependent) set of variables $x$ consisting of voltage magnitude and phase at $\mathrm{AC}$ nodes, voltage at $\mathrm{DC}$ nodes as well as power flows over $\mathrm{AC}$ and DC branches.

Regarding the $N-1$ criterium, the model is able to take into account the outage of a branch (overhead line, power transformer or cable) or a generic equipment inside the system (power generator, power converter). Both the preventive and the corrective $N-1$ methodology can be used. Furthermore, there is no particular restriction about the grid topology. This means that it is possible to solve meshed $\mathrm{AC}$ and DC (e.g. multiterminal DC) synchronous and asynchronous grids.

The mathematical model can be formulated as the following nonlinear optimization problem:

$$
\begin{aligned}
\underset{u_{0}, \ldots u_{N_{c}}}{\operatorname{minimize}} & p_{0} f\left(u_{0}, x_{0}\right)+\sum_{c=1}^{N_{c}} p_{c} f\left(u_{c}, x_{c}\right) \\
\text { subject to } \quad g_{c}\left(u_{c}, x_{c}\right) & =0 \quad c=0 \ldots N_{c}, \\
h_{c}\left(u_{c}, x_{c}\right) & \leq 0 \quad c=0 \ldots N_{c}, \\
\underline{\Delta u_{c}} & \leq u_{c}-u_{0} \leq \overline{\Delta u_{c}},
\end{aligned}
$$

where $N_{c}$ is the number of contingency cases considered ( $c=0$ corresponds to the base case where no contingencies have happened). The set of equality constraints $g_{c}\left(u_{c}, x_{c}\right)$ describes the power balance for each node and equipment, while the set of inequality constraints $h_{c}\left(u_{c}, x_{c}\right)$ describes the physical limits of each component.

The deviation of the control variable in case of corrective $N-1$ method can be accordingly limited with the upper $\overline{\Delta u_{c}}$ and lower $\Delta u_{c}$ bounds (e.g. active power setpoint deviation between the contingency cases and the base case).

The objective function $f$ may be chosen as the generator dispatch cost in the system, system losses, voltage magnitude or angle variability, reactive power generated or another power system objective. It is also possible to provide flexible weights for the different contingency cases under consideration. In this work the objective function is defined as the economic dispatch cost in quadratic form:

$$
\begin{aligned}
\zeta=\sum_{g=1}^{N_{g}}\left\{\alpha_{g}+\beta_{g} P_{g}+\gamma_{g} P_{g}^{2}\right\} \\
+\sum_{c=1}^{N_{c}}\left\{\sum_{g=1}^{N_{g}}\left\{c_{g}^{+} \Delta p_{g, c}^{+}+c_{g}^{-} \Delta p_{g, c}^{-}\right\}\right\}
\end{aligned}
$$


Here $N_{g}$ is the number of generators in the grid, $P_{g}$ is the active power generated by the generator $g$, while $\alpha_{g}, \beta_{g}, \gamma_{g}$ are cost coefficients for the individual generators. $\Delta p_{g, c}^{+}$and $\Delta p_{g, c}^{-}$are the active power deviation (positive and negative) for each contingency and they are calculated with respect to the base case and weighed with the relative linear cost coefficients $c_{g}^{+}$and $c_{g}^{-}$.

The grid model is composed of the following items: nodes (AC and DC), power loads, branches and active equipments (generators, converters and reactive power sources like e.g. static VAR compensators (SVC)). Two power balance equations are given for the $\mathrm{AC}$ nodes and one for the $\mathrm{DC}$ nodes. In the subsequent paragraphs an overview over the models of the grid components is provided.

The following equation describes the active power balance for an $\mathrm{AC}$ node:

$$
-p\left(v_{n, c}, \theta_{n, c}\right)+p_{g, c}+p_{h, a c, c}-p_{n, d}=0,
$$

where the subscripts $n$ and $c$ denote the $n$-th node and the $c$-th contingency case. The term $p\left(v_{n, c}, \theta_{n, c}\right)$ is the active power flow into the $n$-th AC node where $v$ and $\theta$ are the voltage magnitude and phase; the term $p_{g, c}$ is the power supplied by the $g$-th active source; the term $p_{h, a c, c}$ is the power supplied by the $h$-th converter; the term $p_{d}$ is the power demand. In the same way it is possible to describe the reactive power balance for the AC nodes:

$$
-q\left(v_{n, c}, \theta_{n, c}\right)+q_{g, c}+q_{h, a c, c}-q_{n, d}=0 .
$$

For the DC nodes, only one power balance equation is needed:

$$
-p\left(v_{n, c}\right)+p_{h, d c, c}=0,
$$

since in this case no power generators and loads are present. For both AC and DC nodes the voltage magnitude is constrained to its lower and upper limits.

The branches are modeled as generic passive components able to transfer current from one node to another according to Kirchhoff's circuit laws. The double bi-pole model with PI scheme has been used. This model can be applied to overhead lines, cables, power transformers (with and without tap changer and/or phase shifter) and to DC overhead lines and cables. Without loss of generality we assume the tap changer and phase shifter to be installed on the from-node of the branch and constrained with their limits. Both can be modelled with a pure gain $\delta$ and a complex rotation $e^{j \theta}$. For a generic branch $b$, contingency $c$ and defining $i$ as from-node and $j$ as to-node, it is possible to describe the relationship between the current and voltage phasors as follows:

$$
\left[\begin{array}{c}
i_{i} \\
i_{j}
\end{array}\right]-\left[\begin{array}{cc}
\overline{y_{i i}} \delta_{b, c}^{2} & \overline{y_{i j}} \delta_{b, c} e^{j \theta_{b, c}} \\
\overline{y_{j i}} \delta_{b, c} e^{-j \theta_{b, c}} & \overline{y_{j j}}
\end{array}\right]\left[\begin{array}{c}
\dot{v}_{i} \\
\dot{v_{j}}
\end{array}\right]=0 .
$$


Exploiting the symmetry of the model, the matrix component with subscript $i i$ is equal to the component with $j j$ and, in the same way, the component with $i j$ is equal to the component with $j i$. Those values arise from the longitudinal and transversal susceptance and conductance parameters. The equations that describe a double bi-pole model are used to model the active and reactive power flow through the branches, and thus those flows are used inside the nodes power balance equations (3), (4), (5). Obviously, the power flow (active power or apparent power or current module) can be constrained at both sides of the branch with an upper limit.

The power generators are generic active components able to inject active and/or reactive power into the system. The generator capability is defined as a domain created by the intersection of a rectangle (active and reactive upper and lower bounds) and a circle (apparent power bound). Additional linear bounds between active and reactive power can be defined in order to customize the capability domain.

In a similar way, by neglecting the active power, voltage controlling reactive power sources can be used inside the model: in this case, a linear relationship between voltage magnitude and reactive power output is used.

The power converters are able to transfer power in both directions between AC and DC nodes. The converter model takes into account the power balance between the AC and DC ports with the losses due to its power electronics. Moreover, the converters may have capability domains described in the same way as for generators. The converter power balance equation is written as follows:

$$
p_{h, a c, c}+p_{h, d c, c}+p_{h, l o s s, c}=0,
$$

where the subscripts los $s$ denotes the power losses inside the $h$-th converter and the $c$-th contingency. The AC side current is determined by the following equation that describes the power balance in the AC converter side:

$$
\frac{\sqrt{p_{h, a c, c}^{2}+q_{h, c}^{2}}}{\sqrt{3} v_{h, a c, c}}-i_{h, a c, c}=0 .
$$

Finally, the power losses are calculated by a formula that creates a quadratic relationship between the current $i_{h, a c, c}$ and losses $p_{h, l o s s, c}$ (see e.g. [11] and [12]) with parameters $\alpha_{h}, \beta_{h}$ and $\gamma_{h}$ that may be individual for each converter:

$$
\alpha_{h}+\beta_{h} i_{h, a c, c}+\gamma_{h} i_{h, a c, c}^{2}-p_{h, l o s s, c}=0 .
$$

This model is suited for steady-state analyses, so that transient behaviour, e.g. fault and stability analysis cannot be investigated with it. 


\section{Case Study}

The case study in this work is based on the IEEE118 test grid (see [13]).

The generator costs have been modified in order to encourage power transfer from the (low cost) western zone to the (high cost) eastern zone (see Fig. 1). In particular, the generation cost in the western zone have been set to zero to emulate renewable generation, while the cost coefficients in the western zone have not been modified.

The focus of our analysis lies on one additional power corridor between the nodes 8 and 65 (highlighted in red in Fig. 1). There are three options for this considered corridor: an AC corridor, a monopolar HVDC corridor as well as a bipolar HVDC corridor with identical total power ratings of 500 MVA and total length of $500 \mathrm{~km}$. The electrical parameters of the AC corridor as well as the resistance of the DC lines are taken from [14]. Due to the huge length of the corridor the AC line has been split in three line segments. The HVDC converters exhibit a loss model with a quadratic relationship to the AC current (see [15]).

For all three configurations the effects of security constraints on the corridor are analysed. For both the AC corridor and the monopolar HVDC corridor the considered contingency implies the outage of the whole corridor, while for the bipolar configuration an $N-1$ contingency case involved the outage of one of the two poles.

In order to discourage redispatch of generators in the contingency case, we require the generator active and reactive power output to be identical in the base case and the contingency case, while HVDC converter setpoints may differ between base case and contingency case. This means that the generators follow a preventive security strategy while HVDC converters follow a corrective security strategy. The same behaviour can be achieved by imposing very high redispatch cost on the generators. The possibly changing losses after an outage are balanced by adapted voltage profiles. In addition, the setpoints for transformers are fixed.

\section{Implementation}

A software tool for solving the security-constrained optimal power flow problem as described in Sect. 2 has been developed in C++ using Microsoft ${ }^{\circledR}$ Visual Studio ${ }^{\mathrm{TM}}$. Inside this software the open-source library IPOPT (see [16]) is used as solver for non-linear programming based on an interior point method. To speed up the optimization process and to increase accuracy, we derived the analytic forms of Jacobian and Hessian matrices of the objective function and the constraints and pass them directly to the solver instead of using numerical differentiation.

The input data as well as the optimization results including all control and state variables are given as text files. In the results discussion below (Sect. 5) we summarize these results in the form of the key performance indicators dispatch 


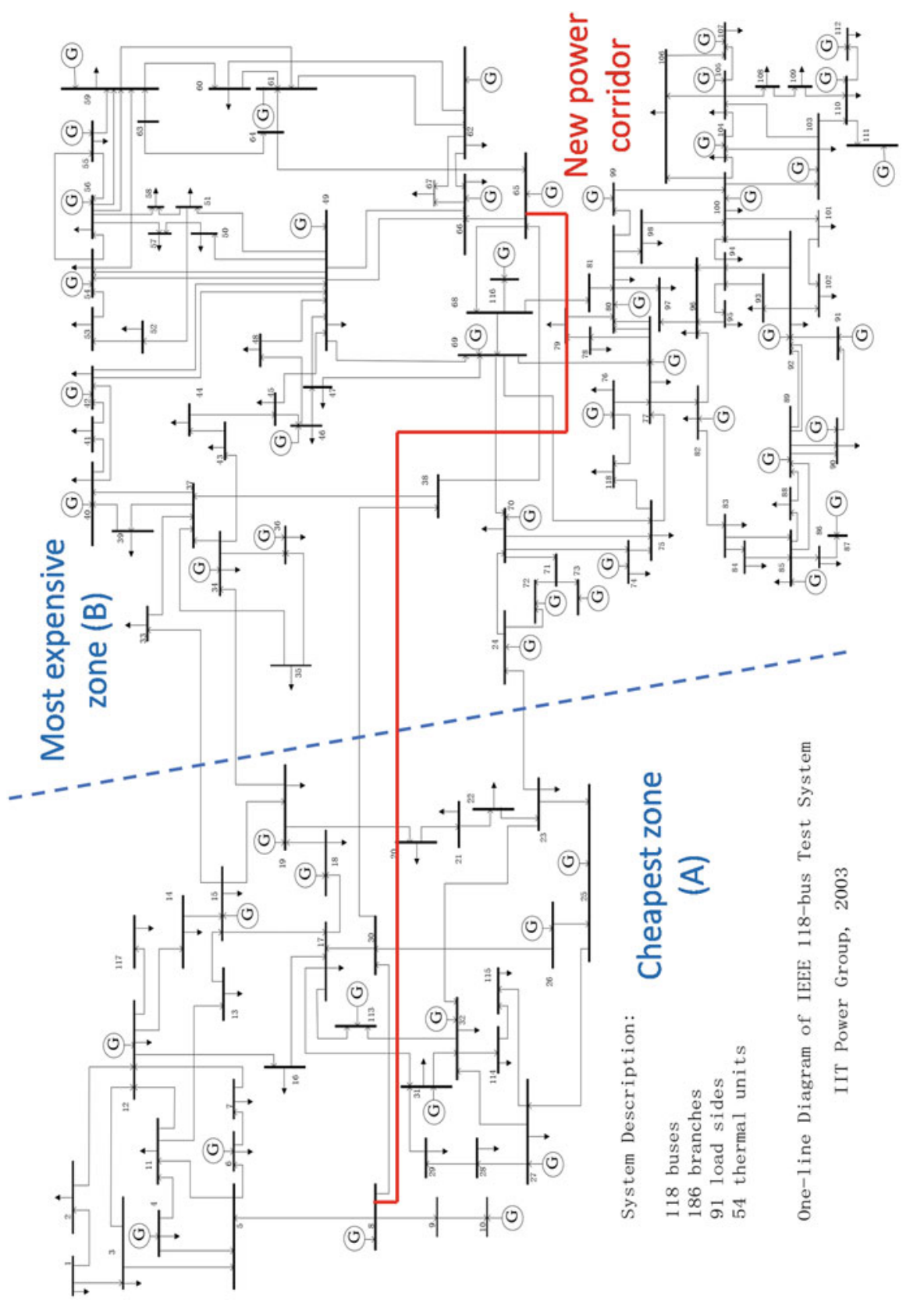


cost, power flow over the considered corridor, active power losses and voltage angle deviation.

\section{Results}

The result of the SC-OPF is analysed in terms of dispatch cost (Fig. 2), power flow over the considered corridor (Fig. 3), active power losses in the system (Fig. 4) and voltage angle difference along the corridor (Fig. 5).

The first observation is that in the cases without the $N-1$ criterion, all the values for the monopolar and the bipolar HVDC corridors coincide, which is obvious since they have identical parameters and rating. When looking at the dispatch cost in Fig. 2 it can be observed that the cost is lowest for both the HVDC variants without $N-1$ criterion. With $N-1$ criterion both HVDC variants have lower dispatch cost than the AC variant, while the bipolar configuration has the lowest dispatch cost. This can be explained by the higher efficiency of the HVDC systems compared to the AC corridor (lower losses over long distances) and the higher redundancy of the bipolar configuration compared to the monopolar one. This way more power can

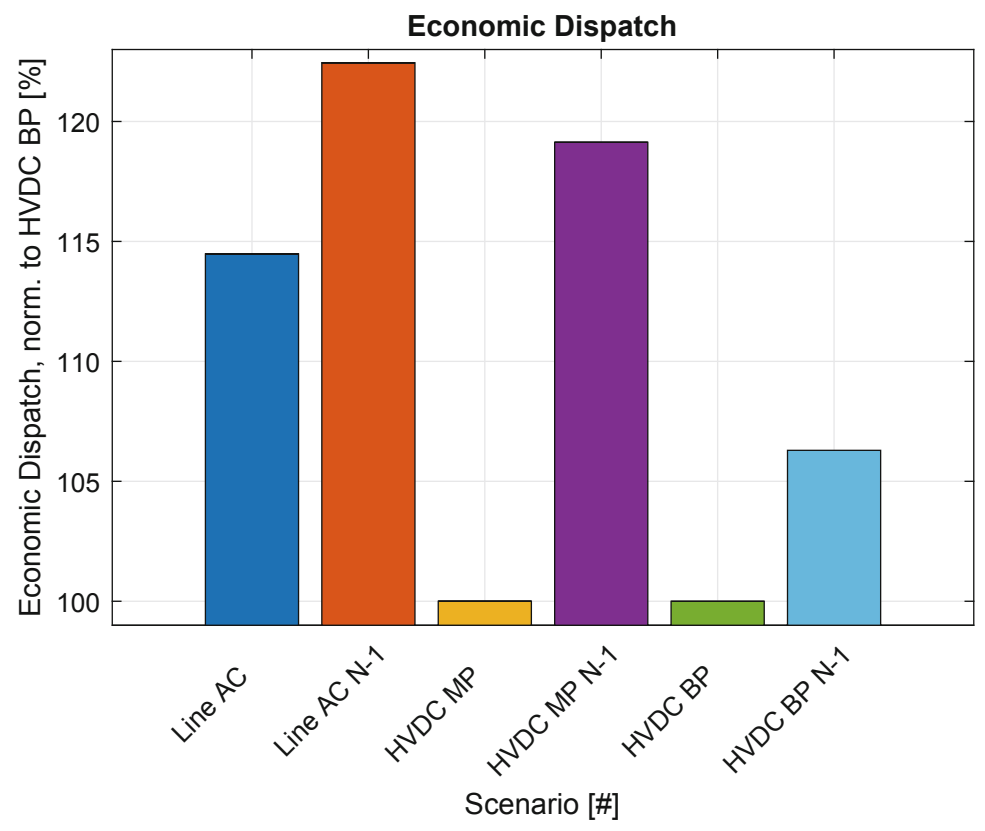

Fig. 2 Dispatch cost relative to bipolar HVDC scenario without $N-1$. Red, dark blue: AC line with/without $N-1$; violet, yellow: monopolar HVDC with/without $N-1$; light blue, green: bipolar HVDC with/without $N-1$ 


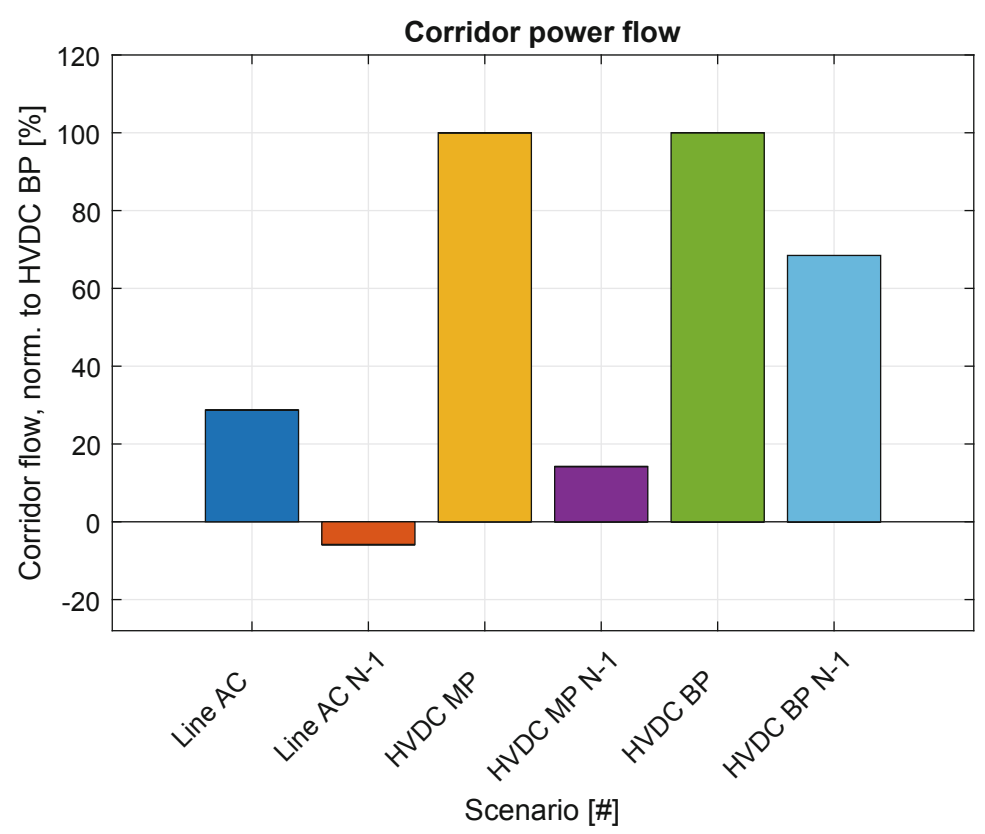

Fig. 3 Power flow over corridor relative to bipolar HVDC scenario without $N-1$ (457 MW)

be transferred from the low cost generation zone to the high cost generation zone which enables a generation dispatch at lower total cost.

This explanation is confirmed by the power flow over the corridor (Fig. 3), where the bipolar HVDC configuration is able to transfer the most power from the low cost generation zone to the high cost generation zone. The monopolar HVDC corridor is transferring significantly less power in the $N-1$ case since it has to prepare for the outage of the full corridor. The AC corridor is not even able to maintain the power flow direction in the $N-1$ case. The significant deviations in the power flows between the AC and monopolar HVDC configuration can be explained by voltage limits and differences in the generator dispatch.

The active power losses in the system can be seen in Fig. 4. There we did not observe a clear pattern, which can be explained by the fact that in all the scenarios the generator dispatch and thus the power flows through the whole system are very different, and the losses were not the objective of the optimization performed.

When looking at the angle deviations in Fig. 5 is can be observed that the AC corridor exhibits a large angle deviation in the case without security criterion, while all the HVDC configurations are able to maintain smaller angle deviations which indicates a higher system stability.

In Table 1 the voltage magnitudes and reactive power injected at the corridor terminal nodes 8 and 65 are listed for the $N-1$ scenario before and after the contingencies. It can be observed that the bipolar HVDC configuration is able to 


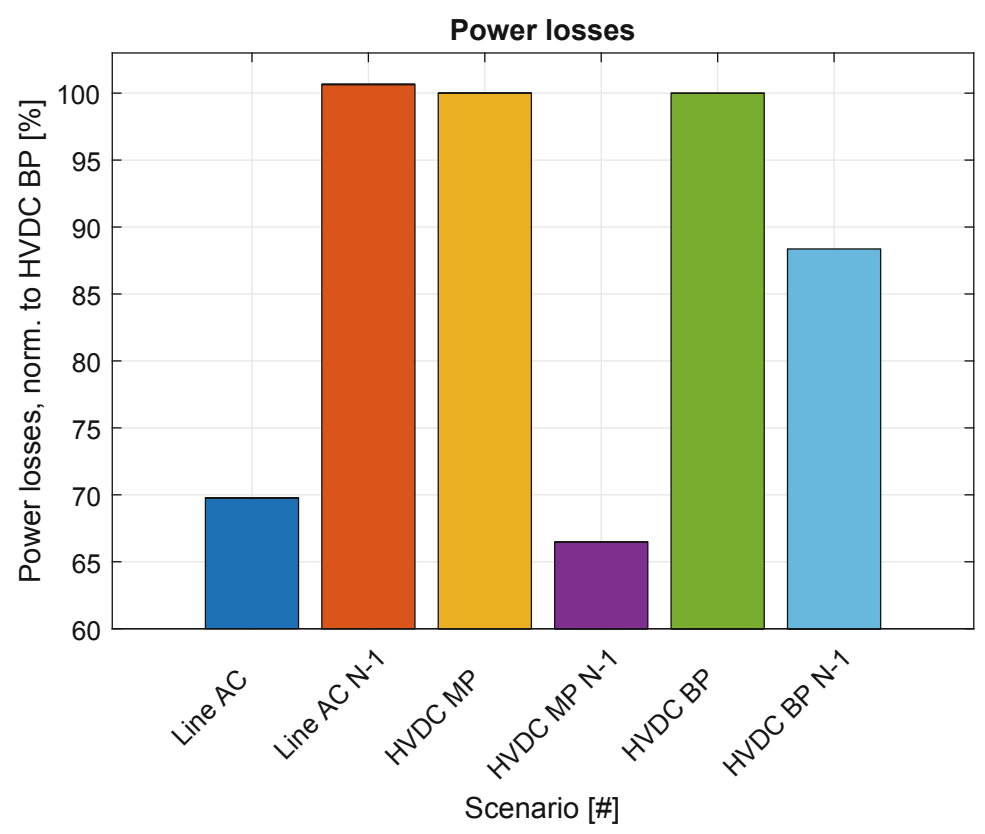

Fig. 4 Active power losses in the system relative to bipolar HVDC scenario without $N-1$ (120 MW)

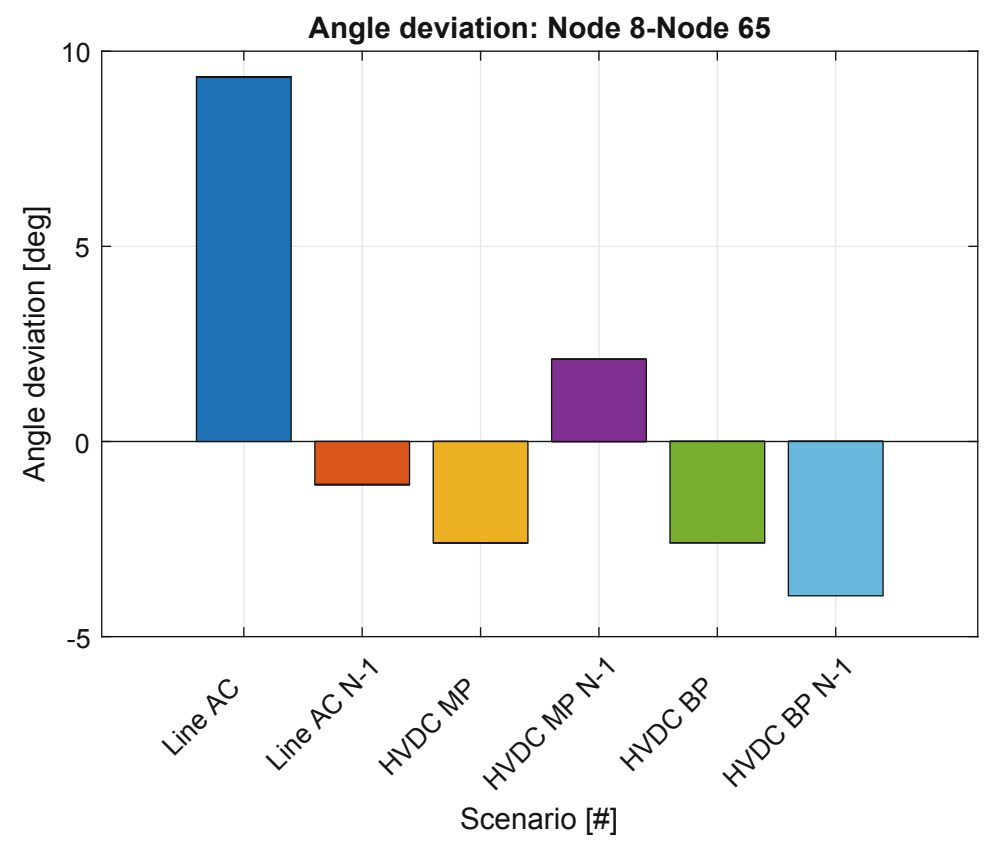

Fig. 5 Angle Deviation over corridor in degrees 
Table 1 Voltage magnitude and reactive power injection at nodes 8 and 65 for all three corridor configurations in the base case and the contingency case of the $N-1$ scenarios

\begin{tabular}{l|l|l|r|r}
\hline Case & $\begin{array}{l}\mathrm{V}(8) \\
\text { [p.u.] }\end{array}$ & $\begin{array}{l}\mathrm{V}(65) \\
\text { [p.u.] }\end{array}$ & $\begin{array}{l}\mathrm{Q}(8) \\
\text { [MVAr] }\end{array}$ & $\begin{array}{l}\mathrm{Q}(65) \\
\text { [MVAr] }\end{array}$ \\
\hline AC; base & 0.980 & 0.968 & 98.2 & 118.6 \\
\hline AC, cont. & 1.046 & 0.965 & 0.0 & 0.0 \\
\hline DC MP; base & 1.039 & 1.012 & -19.0 & -96.1 \\
\hline DC MP, cont. & 0.973 & 1.021 & 0.0 & 0.0 \\
\hline DC BP; base & 1.004 & 1.023 & -58.7 & -5.1 \\
\hline DC BP, cont. & 0.998 & 1.023 & -56.5 & 0.0 \\
\hline
\end{tabular}

maintain a stable voltage level close to the nominal voltage by providing reactive power to the grid. In contrast, for the AC configuration the voltage magnitude at node 8 changes significantly because of high generation in the region. The monopolar HVDC configuration is also stabilizing the voltage by reactive power injection but in the outage case there is the same effect as for the AC corridor.

\section{Conclusion and Future Work}

A mathematical model and a software for security-constrained optimal power flow for generic hybrid AC/DC grids has been developed. In this work it has been used to study the effects of the $N-1$ criterion on embedded HVDC systems.

The above results show that for the bipolar HVDC configuration the power plant dispatch with lowest cost could be established when the $N-1$ criterion is employed. This study has been performed on the IEEE118 grid with a single corridor under consideration. The results indicate the benefits of employing bipolar HVDC systems in order to increase the social welfare.

In order to draw conclusions for real transmission systems, additional studies on real grid configurations with possibly several embedded HVDC links would have to be performed.

Additionally, an investigation including contingency cases both in the AC and DC parts of the power system would be interesting to investigate the capabilities of embedded HVDC to compensate for general $N-1$ cases and thus to reduce redispatch cost for grid operators.

Acknowledgements This research was partly supported by the Federal Ministry of Education and Research (BMBF) within the framework of the project "Neue EnergieNetzStruktURen für die Energiewende"I ENSURE (FKZ 03SFK1N0). 


\section{References}

1. R. Wiget, Combined AC and Multi-terminal HVDC Grids - Optimal Power Flow Formulations and Dynamic Control, Dissertation thesis, ETH Zurich, 2015

2. Bundesnetzagentur, Bestätigung Netzentwicklungsplan Strom, 2017

3. H. Dommel, W. Tinney, Optimal power flow solutions. IEEE Trans. Power Syst. PAS-87(10), 1866-1876 (1968)

4. O. Alsac, B. Stott, Optimal load flow with steady-state security. IEEE Trans. Power Syst. PAS93(3), 745-751 (1974)

5. Network Code on Operational Security, Entso-E Tech. Rep., Sept 2013

6. F. Capitanescu, J.L. Martinez Ramos, P. Panciatici, D. Kirschen, A. Marano Marcolini, L. Platbrood, L. Wehenkel, State-of the-art, challenges, and future trends in security constrained optimal power flow. Electr. Power Syst. Res. 81(8), 1731-1741 (2011)

7. G. Papaefthymiou et al., Methods for optimization of power transits": toolbox for Common Forecasting, Risk assessment, and Operational Optimization in Grid Security Cooperations of Transmission System Operators (TSOs): toolbox for Common Forecasting, Risk assessment, and Operational Optimization in Grid Security Cooperations of Transmission System Operators (TSOs), 2015, Available: https://www.research-collection.ethz.ch/handle/20.500.11850/ 114121. Accessed on 11 Dec 2018

8. S. Chatzivasileiadis, Power System Planning and Operation Methods Integrating the Controllability of HVDC, Dissertation thesis, ETH Zurich, 2013

9. R. Wiget, G. Andersson, Optimal Power Flow for Combined AC and Multi-Terminal HVDC Grids based on VSC Converters, IEEE Power Energy Society (PES) General Meeting, San Diego, 22-26 July 2012

10. N. Meyer-Huebner, S. Weck,F. Bennewitz, M. Suriyah, K. Bhalodi, M. Giuntoli, V. Biagini, A. Krontiris, A. Wasserab, M. Ndreko, M. Wiest, T. Leibfried, J. Hanson, N-1-Secure Dispatch Strategies of Embedded HVDC Using Optimal Power Flow, IEEE Power Energy Society (PES) General Meeting, Portland, 5-10, Aug, 2018, pp. 1-8

11. D. Var Hertem, O. Gomis-Bellmnt, H. Liang, HVDC Grids for Offshore and Supergrids of the Future (IEEE Press/Wiley, New Jersey, 2016), pp. 300-301

12. G. Daelemans, K. Srivastava, M. Reza, S. Cole, R. Belmans, Minimization of steady-state losses in meshed networks using VSC HVDC, IEEE Power Energy Society (PES) General Meeting, Alberta, 26-30 July 2009, pp. 1-5

13. www.ee.washington.edu, IEEE 118 test grid, Available: http://www.ee.washington.edu/ research/pstca/. Accessed on 05 May 2018

14. A.P. Melipoulos, Power System Modeling Analysis and Control, chapter 6 (Marcel Dekker Inc, Atlanta, 2004)

15. D. Var Hertem, O. Gomis-Bellmnt, H. Liang, HVDC Grids for offshore and supergrids of the future (IEEE Press/Wiley, New Jersey, 2016), pp. 300-301

16. A. Wächter, L.T. Biegler, On the implementation of an interior-point filter line-search algorithm for large-scale nonlinear programming. Math. Program. 106(1), 25-57 (2006)

17. A.R. Al-Roomi, Power Flow Test Systems Repository (Electrical and Computer Engineering, Dalhousie University, Halifax, 2015). https://al-roomi.org/power-flow 
Open Access This chapter is licensed under the terms of the Creative Commons Attribution 4.0 International License (http://creativecommons.org/licenses/by/4.0/), which permits use, sharing, adaptation, distribution and reproduction in any medium or format, as long as you give appropriate credit to the original author(s) and the source, provide a link to the Creative Commons licence and indicate if changes were made.

The images or other third party material in this chapter are included in the chapter's Creative Commons licence, unless indicated otherwise in a credit line to the material. If material is not included in the chapter's Creative Commons licence and your intended use is not permitted by statutory regulation or exceeds the permitted use, you will need to obtain permission directly from the copyright holder. 